\title{
High R.E.N.A.L. nephrometry score is associated with partial to total nephrectomy conversion, in treatment of renal cancer
}

\author{
Soares $\mathrm{JP}^{1 *}$, Costa-Matos $\mathrm{A}^{2}$, Toledo LGM${ }^{3}$, Juveniz $\mathrm{J}^{2}$ and Dall Oglio $\mathrm{M}^{2}$ \\ ${ }^{1}$ Faculdade de Tecnologia e Ciências (FTC), Salvador, Brazil \\ ${ }^{2}$ Instituto do Câncer do Estado de São Paulo (ICESP), São Paulo, Brazil \\ ${ }^{3}$ Santa Casa de São Paulo, São Paulo, Brazil
}

\begin{abstract}
Aim: To test the hypothesis that R.E.N.A.L. nephrometry score is able to predict surgical conversion, from laparoscopic to open nephrectomy, or totalization, from partial to total nephrectomy.

Methods: Between January 2010 and June 2012, 320 patients underwent radical or partial nephrectomy at a Tertiary Institution, of which 173 (54.1\%) had a tumor $<7 \mathrm{~cm}$. Of these, $71(41 \%)$ patients were selected for the prospective study according to inclusion and exclusion criteria. Score's accuracy in predicting surgical conversion rate was analyzed by using ROC curve and it's best cutoff point was identified.

Results: The accuracy of R.E.N.A.L. nephrometry score in predicting conversion rate demonstrated an AUC of 0.715 (95\% CI: 0.595-0.836; $\mathrm{p}=0.002$ ). By evaluating a cutoff point, it was found $46 \%$ sensitivity and $78 \%$ specificity for R.E.N.A.L. >9. From patients who underwent totalization, 4/46 (8.9\%) had a R.E.N.A.L. score $<9$ and $7 / 25$ (28\%) had a R.E.N.A.L. score $\geq 9$, OR 4.08, $p=0.03$.

Conclusion: R.E.N.A.L. nephrometry score can help predicting the need of total nephrectomy or conversion to open surgery. Renal unit loss was associated with high complexity tumors (score $\geq 9$ ), with this cutoff point representing a fourfold higher chance of conversion from partial nephrectomy to radical in treatment of renal cancer.
\end{abstract}

\section{Introduction}

Renal cell carcinoma (RCC) represents the most common type of kidney cancer and its incidence is increasing worldwide, also as a reflection of imaging methods diffusion that is capable of early diagnostics [1]. Surgical removal of the tumor is still the only curative treatment for localized renal cancer, with two possible approaches: total or partial nephrectomy, both of which can be performed either openly or laparoscopically.

Over the last two decades there has been a significant expansion of partial nephrectomy (PN) [2]. PN has been established with the purpose of promoting better renal function preservation, prevention of chronic postoperative kidney disease, providing improvement in cardiovascular function and decreasing mortality when compared to total nephrectomy (TN) [3]. Thus, nephron sparing surgery is considered the gold standard for treatment of most tumors up to $4 \mathrm{~cm}$ (T1a), and is an emerging option for some tumors between 4 and $7 \mathrm{~cm}$ (T1b), with oncologic equivalence comparable to TN [4]. For individuals with a single, anatomical or functional kidney, the benefits are clear, allowing this approach to be performed even for more complex cases, such as tumors larger than $7 \mathrm{~cm}$ or multiple ones [5]. However, even individuals with normal renal function and contralateral disease-free kidney, several studies have demonstrated long-term partial approach advantages in preserving renal function [6-11].

Recent improvement in laparoscopic techniques, and the availability of vascular instruments for this purpose, have allowed laparoscopic partial nephrectomy (LPN) to become a viable alternative. LPN requires less analgesic use, shorter hospital stay and better postoperative recovery with satisfactory renal preservation and with great oncological outcomes reported in 5 years [12]. On the other hand, perioperative morbidity and, in some cases, technical difficulty, are usually higher in LPN, making these patients have a higher risk of complications [13].

During surgical approach, sometimes totalization is needed because of some variables that can hinder surgical procedure. Conversion of PN to $\mathrm{TN}$, however, can be predicted if specific tumor parameters are taken into account. R.E.N.A.L. nephrometry score is based on the five main aspects that characterize anatomical attributes of solid renal mass: tumor size, relation to renal cortex (endophytic or exophytic), proximity to excretory system or renal sinus, anterior or posterior localization, and position of the tumor in relation to polar line. It thus represents a structured, reproducible and quantitative system capable of classify and compare tumors according to their anatomical complexity [14].

Correspondence to: Jéssica Palma Soares, Rua Doutor Pedro de Souza Pondé, 09 Salvador, BA, Brazil, ZIP Code 40155-270, ORCID: 0000-0002-0628-3502; Tel: +55-71-98182-8313; E-mail: jeupalma@gmail.com

Key words: nephrectomy oncology, anatomy, kidney

Received: February 27, 2018; Accepted: March 09, 2018; Published: March 14 2018 
Because of the increasing frequency of sparing surgery worldwide, the evaluation of anatomical parameters and the attempt to predict whether they suggest intraoperative totalization or conversion is relevant for surgical and anesthetic planning. Then, the present study intends to test the hypothesis that R.E.N.A.L. nephrometry score is capable of predicting surgical conversion from laparoscopic to open approach, or totalization, from partial to radical nephrectomy.

\section{Materials and methods}

This is a "post hoc" analysis of a prospective cohort study whose data collection was performed between January 2010 and June 2012 with patients admitted in a reference oncologic hospital with indication of renal tumor surgical excision. Patients who accepted to participate in study needed a computed tomography (CT) scan of Hospital service, which was the only intervention performed, essential for the adequacy of R.E.N.A.L. score standardization.

Inclusion criteria used were solid or cystic lesion (Bosniak III or IV) with contrast uptake in kidney with indication of partial nephrectomy: tumor up to $7 \mathrm{~cm}$ in diameter, regardless of anatomical location; to have a tomography or magnetic resonance image in hospital's internal system, allowing complete visualization of images in axial, sagittal and coronal sections; and to be over 18 years old, or younger than 18 with consent of legal tutors. Exclusion criteria were patients with: single anatomic or functional kidney; chronic renal failure (creatinine $\geq 1.5 \mathrm{mg} / \mathrm{dl}$ ); multiple renal tumors (more than one); nephrolitiasis ipsilateral to the tumor; renal tuberculosis; patients submitted to previous surgeries in the kidney or upper abdomen ipsilateral to renal tumor; and those who did not agree to sign consent form.

The following variables were studied: age, sex, BMI (body mass index), systemic arterial hypertension, diabetes mellitus, presence of tumor related symptoms (pain, hematuria, palpable mass, paraneoplastic syndrome), presence of metastasis, anatomopathological data, surgical margin and surgical approach. For the perioperative outcomes, the conversion rate for open surgery and totalization and the respective reasons were considered.

Initially, all patients were submitted to laparoscopic partial nephrectomy, considered the gold standard of approach in this research protocol. Any different approach was considered as conversion. After surgery, patients were classified according to the operation performed: laparoscopic partial nephrectomy (LPN); open partial nephrectomy (OPN); laparoscopic total nephrectomy (LTN); or open total nephrectomy (OTN). Preoperative (preemptive) conversions were those that occurred prior to the beginning of the procedure and intraoperative were those that occurred during the surgery.

Statistical analyzes were performed using chi-square test for comparison between proportions and correction for continuity or Fisher's exact test, when applied. Relative risks estimated were given by Mantel-Haenszel analysis. Receiver Operating Characteristic (ROC) curve was designed to evaluate the accuracy of R.E.N.A.L. score into predicting the rate of conversion of laparoscopic to open access, and Youden index to identify the cutoff point with best performance in sensitivity and specificity. Values of $\mathrm{p}<0.05$ were considered statistically significant.

\section{Results}

Between January 2010 and June 2012, 320 individuals underwent nephrectomy due to RCC. Of these, 173 (54\%) patients had tumors up to $7 \mathrm{~cm}$. Seventy-one patients were included in data analysis, according to selection criteria. The reasons for exclusion were: single kidney (8), chronic renal failure (17), renal tuberculosis (3), pyelonephritis (1), previous renal surgery (7), previous abdominal surgery (11), ipsilateral kidney stone (12), and absence of a CT scan in the hospital internal system (43).

Regarding clinical characteristics, the mean age was $60.0+12.7$ years and 39 (54.9\%) were male. The fact that the tumor was diagnosed incidentally, with no related symptoms, in $76.1 \%$ of patients, is important to highlight. Other characteristics are listed in Table 1.

Accessing anatomical characteristics of the tumors, the mean tumor size was $4.1 \mathrm{~cm}$. Median R.E.N.A.L. score was 9 (4-11). There were 7 (9.9\%) patients of low complexity, 22 (31\%) of high complexity and the majority (59.2\%) of medium complexity. Most frequent pathological stage and histological type were T1a (54.9\%) and clear cell (47.9\%), respectively.

In reference of surgical access route, 40 (56.4\%) patients were operated by laparoscopy (Tables 2 and 3). Most tumors of low

Table 1. Demographic and clinical characteristics of the study population.

\begin{tabular}{|l|l|}
\hline Characteristic & N (\%) \\
\hline Male & $39(55 \%)$ \\
\hline Age & $60 \pm 12.7^{*}$ \\
\hline BMI & $27.6 \pm 4.5^{*}$ \\
\hline ASA I & $5(7 \%)$ \\
\hline ASA II & $60(84 \%)$ \\
\hline ASA III & $6(8.5 \%)$ \\
\hline Systemic Arterial Hypertension & $32(45 \%)$ \\
\hline Diabetes Mellitus & $9(13 \%)$ \\
\hline Incidental & $54(76 \%)$ \\
\hline
\end{tabular}

$*$ Average \pm Standard Deviation (minimum-maximum).

Table 2. Anatomical and pathological characteristics of the operated tumors.

\begin{tabular}{|l|l|}
\hline Characteristic & N (\%) \\
\hline Tumor size & $4.1 \pm 1.4^{*}$ \\
\hline RENAL score & $9(4-11)^{\dagger}$ \\
\hline RENAL score & \\
\hline Low complexity (4-6) & $7(9.9 \%)$ \\
Medium complexity (7-9) & $42(59 \%)$ \\
High complexity (10-12) & $22(31 \%)$ \\
\hline Histological type & \\
Clear cell & $34(48 \%)$ \\
Papillary & $9(13 \%)$ \\
Chromophobe & $6(8.5 \%)$ \\
Medullary & $4(5.6 \%)$ \\
Oncocytoma & $2(2.8 \%)$ \\
Angiomyolipoma & $6(8.5 \%)$ \\
Others & $10(14 \%)$ \\
\hline Pathological stage & \\
T1a & $39(55 \%)$ \\
T1b & $21(30 \%)$ \\
T2 & $6(8.5 \%)$ \\
T3a & $5(7.0 \%)$ \\
\hline Surgical margin study & \\
Free & $50(70 \%)$ \\
Coincidents & $17(24 \%)$ \\
Focused & $2(2.8 \%)-$ laparoscopic \\
Compromised & $2(2.8 \%)-$ open 1 / laparoscopic 1 \\
\hline &
\end{tabular}

$*$ Average \pm Standard Deviation. $\dagger$ Medium (minimum - maximum).

Table 3. Surgical access route.

\begin{tabular}{|l|l|}
\hline Access route & N (\%) \\
\hline Laparoscopic partial nephrectomy & $32(45 \%)$ \\
\hline Open partial nephrectomy & $28(39 \%)$ \\
\hline Laparoscopic total nephrectomy & $8(11 \%)$ \\
\hline Open total nephrectomy & $3(4.2 \%)$ \\
\hline
\end{tabular}


complexity (71.4\%), approximately half of the tumors of medium (52.4\%), and the minority of high ones $(22.7 \%)$ were operated by laparoscopic partial nephrectomy $(\mathrm{p}<0.01)$.

Preemptive conversion to open surgery occurred in $26(36.6 \%)$ patients, 2 for OTN and 24 for OPN. Forty-five patients started the procedure by laparoscopy, 8 were converted to LTN and 5 were converted to open surgery (4 OPN and 1 OTN).

In concerning of totalization frequency, 2 (7.7\%) patients who started open surgery and $9(20 \%)$ patients who started with videolaparoscopy were converted to radical nephrectomy $(\mathrm{p}=0.14)$.

The accuracy of R.E.N.A.L. score in predicting conversion rate was tested through the ROC curve which demonstrated an AUC of 0.715 (0.595-0.836), $\mathrm{p}=0.002$ (Figure 1). Evaluating a cut-off point privileging information specificity, $46 \%$ sensitivity and $78 \%$ specificity were found for R.E.N.A.L. $>9$.

Then, patients were separated into two groups, from the cut-off point found by the Youden index, and the association of each group with the types of surgery were verified. Patients with R.E.N.A.L. $<9$ were more frequently submitted to PN (93\% x 72\%, p=0.03) and LPN (54.3\% x 28\%, $\mathrm{p}=0.02)$. Twenty-five patients (54.3\%) with R.E.N.A.L. $<9$ and only seven $(28 \%)$ with R.E.N.A.L. $\geq 9$ underwent LPN without any kind of conversion; $\mathrm{p}=0.03$.

From the individuals that underwent totalization, $4 / 46(8.9 \%)$ had R.E.N.A.L. $<9$ and $7 / 25$ (28\%) had R.E.N.A.L. $\geq 9$ ( $p=0.03)$. The Odds Ratio was 4.08 (1.06-15.7) and the accuracy of R.E.N.A.L. score in predicting totalization was 0.69 , showing $63.6 \%$ sensitivity and $70 \%$ specificity.

\section{Discussion}

In the present study, the analysis of R.E.N.A.L. score capacity of predicting conversion rate and totalization, showed a moderate and low accuracy respectively. Then, the value of 9 was determined as the best cutoff point of the score. After this, two groups were separated

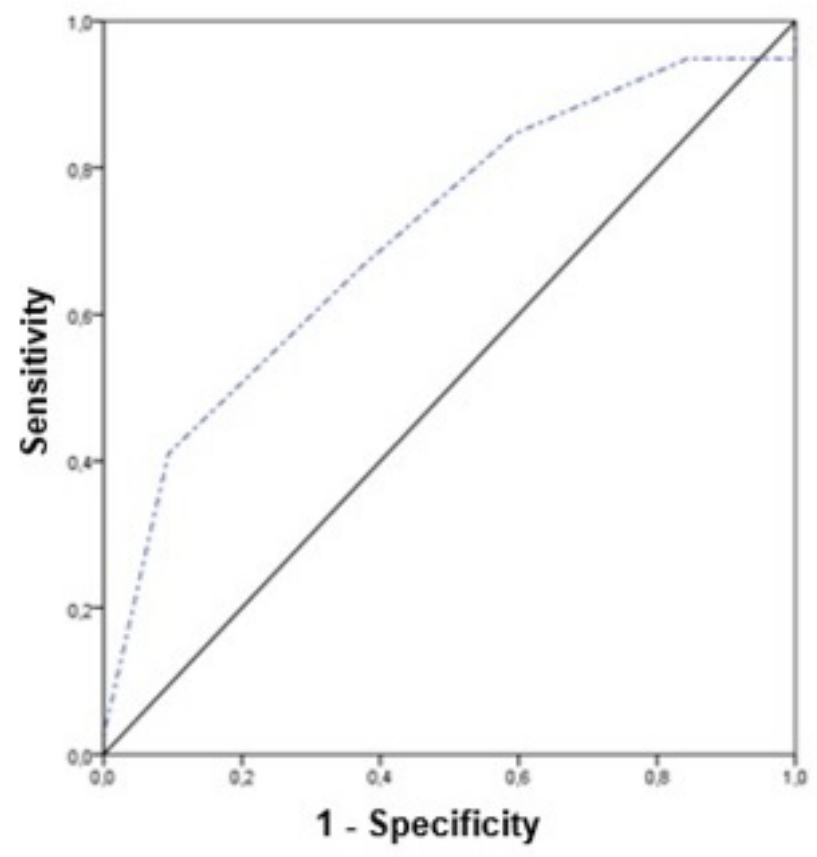

Figure 1. ROC curve: R.E.N.A.L. X Conversion rate. according tumor's complexity and it was found that the tumors of high complexity have four times more chances of renal unit loss. Thus, it was observed R.E.N.A.L. score is associated with conversion of partial into total nephrectomy, regarding treatment of renal cancer.

Definition of anatomical aspects and tumor location by nephrometry is what makes it possible to identify patients with greater chance of conversion/totalization, suggesting its efficacy in determining possible intraoperative findings that hinder the effectiveness of nephron sparing surgery. The use of this instrument, therefore, facilitates the indication of the approach route and in individuals with a score greater than or equal to 9 , who are at high risk, it is possible to predict the need to initiate nephrectomy by open and/or total and thus avoid intraoperative conversion. However, there is a high association, but R.E.N.A.L. score alone does not allow to identify with good accuracy patients who will have these outcomes. In complex models, such as biological ones, multiple variables added together are often needed to predict outcomes.

The knowledge of the increased risk of chronic kidney disease and cardiovascular morbidity after radical nephrectomy, in addition to equivalent oncological outcomes in tumors of different sizes [15], led the European Association of Urology (EAU) and the American Urology Association (AUA) to support the use of sparing surgery whenever technically feasible [16]. Seung et al. presented in their study the increase in frequency of PN from $59.5 \%$ in 2008 to $95.1 \%$ in 2014 in low complexity group, from $23.1 \%$ in 2008 to $75.3 \%$ in 2014 in medium complexity group and from $2.2 \%$ in 2008 to $19.6 \%$ in 2014 in high complexity group [2]. Another evaluation carried out in European tertiary centers found an increase from 15\% to 70\% between 1987 and 2008 [17]. In our sample, the frequency of PN was $84.5 \%, 53 \%$ of which were videolaparoscopy. This result shows the tendency of Brazilian tertiary care centers reproduce the indication of nephron sparing surgery that occurs in other important centers worldwide.

In this study, $7.7 \%$ of patients submitted to OPN and $20 \%$ of those submitted to LPN converted for totalization $(\mathrm{p}=0.14)$. Despite the lack of statistical significance, the literature demonstrates greatest technical difficulty of laparoscopy as a factor that contributes to increase the indication of total nephrectomy [18]. However, it is a consensus among urologists that it is better to perform an open partial nephrectomy than a total laparoscopic one [18]. Even with the benefits already published on general survival, with oncological and functional outcomes similar to the open surgery [16], Gill et al. evidenced the increase in the rate of perioperative complications. The publication showed a three times greater chance of performing secondary procedures in LPN in a study comparing LPN and OPN performed in patients with single tumors smaller than $7 \mathrm{~cm} \mathrm{[3].}$

The decision of surgical indication based on preoperative imaging is often difficult. Therefore, R.E.N.A.L. nephrometry score was designed to describe and classify the most surgically relevant anatomical features of solid renal neoplasms in attempt to minimize differences in standardization of conducts between services [14]. Currently, nephrometry is spreading, being proven effective for several aspects, but it's underutilization still leads to an often intraoperative final decision.

Previous studies have shown a correlation between the score and the surgical decision, oncological and functional results, and postoperative complications [19-24]. In our study, high nephrometry (R.E.N.A.L. $\geq 9$ ) had a $46 \%$ sensitivity and a $78 \%$ specificity in predicting conversion rate and a $63.6 \%$ sensitivity and a $70 \%$ specificity in predicting totalization. There was a $28 \%$ totalization in high complexity group against $8.9 \%$ in lower complexity group, showing a chance of intraoperative 
totalization of 4.08 when RENAL $\geq 9$. In agreement, a study that evaluated the capacity of nefrometry in predicting perioperative results, demonstrated in a series of 134 PNs $23 \%$ of complication for R.E.N.A.L. $\geq 9$, elucidating the possibility of avoiding this risk with a correct indication [25].

Still according to our work, Canter et al. found in a cohort of 615 patients that score could be a valuable tool for objectifying decision making process of surgical indication. Tumors treated by TN had a mean score of 9.67 and those treated by PN of 7.49. Analysis of the individual components showed as the tumor size $(\mathrm{R})$, proximity to the collecting system $(\mathrm{N})$ and location $(\mathrm{L})$ scores increased, TN was the most likely indication [20]. However, most of the published studies do not test accuracy, but only find associations between variables of the score and outcomes. As the data about R.E.N.A.L. score are being validated by studies with larger casuistics, it will be possible to have a better prediction of chances of conversion, improving anesthetic and surgical planning and preparation of the patient for this possibility.

The present study, although prospective, did not control some variables. Variation of the surgical teams may have influenced the choice of access route. Another criticism is that it was a "post hoc" study, so the initial project was not designed to answer these objectives. Anyway, association between outcomes and tumor complexity was clearly established, making R.E.N.A.L. score a useful tool in therapeutic planning of patients with renal cancer.

R.E.N.A.L. nephrometry score can help predict the need for nephrectomy conversion and/or totalization. Renal unit loss was associated with high complexity tumors (score $\geq 9$ ), with this cutoff representing a fourfold higher chance of intraoperative kidney loss in renal cancer treatment.

\section{Author's contribution}

JP Soares: Protocol/project development, Data collection or management, Data analysis and Manuscript writing/editing.

A Costa-Matos: Protocol/project development, Data collection or management, Data analysis and Manuscript writing/editing.

LGM Toledo: Protocol/project development, Data collection or management, Data analysis and Manuscript writing/editing.

J Juveniz: Protocol/project development, Data collection or management, Data analysis and Manuscript writing/editing.

M Dall'Oglio: Protocol/project development, Data collection or management, Data analysis and Manuscript writing/editing.

\section{References}

1. Camacho JC, Kokabi N, Xing M, Master VA, Pattaras JG et al. (2015) R.E.N.A.L. Nephrometry Score Predicts Early Tumor Recurrence and Complications after Percutaneous Ablative Therapies for Renal Cell Carcinoma? A 5-Year Experience. $J$ Vasc Interv Radiol $1-8$.

2. Shin SJ, Ko KJ, Kim TS, Ryoo HS, Sung HH, et al. (2015) Trends in the Use of Nephron-Sparing Surgery over 7 Years: An Analysis Using the R.E.N.A.L. Nephrometry Scoring System. PLoS One 10: e0141709. [Crossref]

3. Gill IS, Kavoussi LR, Lane BR, Blute ML, Babineau D, et al. (2007) Comparison of 1,800 laparoscopic and open partial nephrectomies for single renal tumors. J Urol 178 : 41-46. [Crossref]

4. Leslie S, Gill IS, Luis A, Abreu DC, Rahmanuddin S, Gill KS, et al. (2014) Renal Tumor Contact Surface Area: A Novel Parameter for Predicting Complexity and Outcomes of Partial Nephrectomy. Eur Urol 1-10.[Crossref]

5. Zini L, Patard JJ, Capitanio U, Mejean A, Villers A, et al. (2009) The use of partial nephrectomy in European tertiary care centers. Eur J Surg Oncol 35: 636-642. [Crossref]
6. Matin SF, Gill IS, Worley S, Novick AC (2002) Outcome of laparoscopic radical and open partial nephrectomy for the sporadic $4 \mathrm{~cm}$. or less renal tumor with a normal contralateral kidney. J Urol 168:1356-1360. [Crossref]

7. Huang WC, Levey AS, Serio AM, Snyder M, Vickers AJ, et al. (2006) Chronic kidney disease after nephrectomy in patients with renal cortical tumours: a retrospective cohort study. Lancet Oncol 7:735-740. [Crossref]

8. Thompson RH, Siddiqui S, Lohse CM, Leibovich BC, Russo P, et al. (2009) Partia versus radical nephrectomy for 4 to $7 \mathrm{~cm}$ renal cortical tumors. J Urol 182: 2601-2606. [Crossref]

9. Thompson RH, Siddiqui S, Lohse CM, Leibovich BC, Russo P, et al. (2009) Partial versus radical nephrectomy for 4 to $7 \mathrm{~cm}$ renal cortical tumors. J Urol 182: 2601-2606. [Crossref]

10. Capitanio U, Terrone C, Antonelli A, Minervini A, Volpe A, et al. (2015) Nephronsparing techniques independently decrease the risk of cardiovascular events relative to radical nephrectomy in patients with a $\mathrm{T} 1 \mathrm{a}-\mathrm{T} 1 \mathrm{~b}$ renal mass and normal preoperative renal function. Eur Urol 67: 683-689. [Crossref]

11. Huang WC, Elkin EB, Levey AS, Jang TL, Russo P (2009) Partial Nephrectomy Versus Radical Nephrectomy in Patients With Small Renal Tumors-Is There a Difference in Mortality and Cardiovascular Outcomes? J Urol 181: 55-62.

12. Lane BR, Gill IS (2010) 7-year oncological outcomes after laparoscopic and open partial nephrectomy. J Urol 183: 473-479. [Crossref]

13. Gill IS, Matin SF, Desai MM, Kaouk JH, Steinberg A, et al. (2003) Comparative analysis of laparoscopic versus open partial nephrectomy for renal tumors in 200 patients. J Urol 170: 64-68. [Crossref]

14. Kutikov A, Uzzo RG (2009) The R.E.N.A.L. Nephrometry Score?: A Comprehensive Standardized System for Quantitating Renal Tumor Size, Location and Depth. JURO 182: 844-853. [Crossref]

15. Crépel M, Jeldres C, Sun M, Lughezzani G, Isbarn H, Alasker A, et al. (2010) A Population-based Comparison of Cancer-control Rates Between Radical and Partial Nephrectomy for T1A Renal Cell Carcinoma. Urology 76: 883-888.[Crossref]

16. Smith ZL (2016) Current Status of Minimally Invasive Surgery for Renal Cell Carcinoma. Curr Urol Rep 17: 43. [Crossref]

17. Meyer C, Hansen J, Becker A, Schmid M, Pradel L, et al. (2016) The Adoption of Nephron-Sparing Surgery in Europe - A Trend Analysis in Two Referral Centers from Austria and Germany. Urol Int 96: 330-336. [Crossref]

18. Cozar JM, Tallada M (2008) Open partial nephrectomy in renal cancer: a feasible gold standard technique in all hospitals. Adv Urol. [Crossref]

19. Mufarrij PW, Krane LS, Rajamahanty S, Hemal AK (2011) Does Nephrometry Scoring of Renal Tumors Predict Outcomes in Patients Selected for Robot-Assisted Partial Nephrectomy? J Endourol 25:1649-1653. [Crossref]

20. Canter D, Kutikov A, Manley B, Egleston B, Simhan J, Smaldone M, et al. (2011) Utility of the R.E.N.A.L. Nephrometry Scoring System in Objectifying Treatment Decision-making of the Enhancing Renal Mass. Urology 78: 1089-1094. [Crossref]

21. Hurst FP, Jindal RM, Fletcher JJ, Dharnidharka V, Gorman G, et al. (2011) Anatomic Features of Enhancing Renal Masses Predict Malignant and High-Grade Pathology: A Preoperative Nomogram Using the RENAL Nephrometry Score. J Urol 186: 836-837. [Crossref]

22. Bruner B, Breau RH, Lohse CM, Leibovich BC, Blute ML (2011) Renal nephrometry score is associated with urine leak after partial nephrectomy. BJU Int 108: 67-72. [Crossref]

23. Rosevear HM, Gellhaus PT, Lightfoot AJ, Kresowik TP, Joudi FN, et al. (2011) Utility of the RENAL nephrometry scoring system in the real world?: predicting surgeon operative preference and complication risk. J Urol 700-705. [Crossref]

24. Simhan J, Smaldone MC, Tsai KJ, Canter DJ, Li T, et al. (2011) Objective Measures of Renal Mass Anatomic Com- plexity Predict Rates of Major Complications Following Partial Nephrectomy Eur Urol 63: 94270. [Crossref]

25. Hew MN, Baseskioglu B, Barwari K, Axwijk PH, Can C, et al. (2011) Critical Appraisa of the PADUA Classification and Assessment of the R.E.N.A.L. Nephrometry Score in Patients Undergoing Partial Nephrectomy. J Urol 186: 42-46. [Crossref]

Copyright: (C2018 Soares JP.This is an open-access article distributed under the terms of the Creative Commons Attribution License, which permits unrestricted use, distribution, and reproduction in any medium, provided the original author and source are credited. 